disadvantages compared with modern molecular methods, including a lower sensitivity and greater delay before the result is available [7, 8]. The prompt and accurate detection of a respiratory virus infection in patients with underlying lung disease is important for a number of reasons. Anti-viral medications are sometimes indicated for patients with a suspected or confirmed respiratory viral infection, particularly RSV or influenza [9]. Some respiratory viral infections are associated with increased morbidity and mortality in patients with chronic lung disease [10]. Confirmation of a respiratory viral infection may therefore prompt early institution of close monitoring and supportive measures, or commencement of preemptive antibiotic therapy to prevent clinical deterioration from an accompanying bacterial exacerbation, particularly for those patients with known chronic bacterial infection. In addition, infection control measures may require prompt diagnosis of a respiratory viral infection [3]. For example, since this study ended, a case of pandemic H1N1 influenza was positive by sputum PCR but negative by nasal swab PCR. In some cases, the identification of a respiratory virus by PCR was not confirmed from a nasal swab specimen but was confirmed from an accompanying sputum sample. As many adults with CF and other chronic lung diseases produce sputum during infective exacerbations, this may be an easily available and acceptable specimen to obtain from patients for screening for viral infection, with greater sensitivity than nasal swab samples.

\footnotetext{
A.M. Jones*, , W. Flight*, B. Isalska , M. Cullen , K. Mutton ${ }^{+}$, A. Bowling* , D. Riley*, K. Webb*,\# and R. Bright-Thomas*,\# *Manchester Adult Cystic Fibrosis Centre, "Medical Microbiology, University Hospitals South Manchester NHS Trust, "Respiratory Research Group, Faculty of Medical and Human Sciences, The University of Manchester, Manchester Academic Health Centre, and ${ }^{+}$Dept of Virology, Manchester Royal Infirmary, Manchester, UK.
}

Correspondence: A.M. Jones, Manchester Adult Cystic Fibrosis Centre, University Hospitals South Manchester NHS
Trust, Southmoor Road, Manchester M23 9LT, UK. E-mail: andrew.jones@UHSM.nhs.uk

Statement of Interest: None declared.

\section{REFERENCES}

1 Perez-Padilla R, de la Rosa-Zamboni D, Ponce de Leon S, et al. Pneumonia and respiratory failure from swine-origin influenza A (H1N1) in Mexico. N Engl J Med 2009; 361: 680-689.

2 Booth C, Matukas L, Tomlinson G, et al. Clinical features and short-term outcomes of 144 patients with SARS in the greater Toronto area. JAMA 2003; 289: 2801-2809.

3 British Infection Society, British Thoracic Society, Health Protection Agency. Pandemic flu: clinical management of patients with an influenza-like illness during an influenza pandemic. Provisional guidelines from the British Infection Society, British Thoracic Society, and Health Protection Agency in collaboration with the Department of Health. Thorax 2007; 62: Suppl. 1, 1-46.

4 Johnston S, Sanderson G, Pattemore P, et al. Use of polymerase chain reaction for diagnosis of picornavirus infection in subjects with and without respiratory symptoms. J Clin Microbiol 1993; 31: 111-117.

5 Collinson J, Nicholson K, Cancio E, et al. Effects of upper respiratory tract infections in patients with cystic fibrosis. Thorax 1996; 51: 1115-1122.

6 Punch G, Syrmis MW, Rose BR, et al. Method for detection of respiratory viruses in the sputa of patients with cystic fibrosis. Eur J Clin Microbiol Infect Dis 2005; 24: 54-57.

7 Wat D, Doull I. Respiratory virus infections in cystic fibrosis. Paediatr Respir Rev 2003; 4: 172-177.

8 Clifton IJ, Kastelik JA, Peckham DG, et al. Ten years of viral and non-bacterial serology in adults with cystic fibrosis. Epidemiol Infect 2008; 136: 128-134.

9 Jefferson T, Demicheli V, Rivetti D, et al. Antivirals for influenza in healthy adults: systematic review. Lancet 2006; 367: 303-313.

10 Bhat $\mathrm{N}$, Wright $\mathrm{J}$, Broder $\mathrm{K}$, et al. Influenza-associated deaths among children in the United States, 2003-2004. N Engl J Med 2005; 353: 2559-2567.

\title{
Exacerbations of idiopathic pulmonary fibrosis treated with corticosteroids and cyclophosphamide pulses
}

\section{To the Editors:}

Acute exacerbations (AEs) are now recognised as a frequent and severe complication of idiopathic pulmonary fibrosis (IPF). In a large series, 1- and 3-yr incidences were $14.2 \%$ and $20.7 \%$, respectively [1]. New diagnostic criteria were published in 2007 [2]. The pathogenesis of these episodes remains unknown, although invasive procedures have been described as possible triggers. Prognosis is poor, with a short-term mortality rate of $45-85 \%$. There is no consensus regarding treatment, as no published study has compared the efficiency of different treatment regimens, and treatment often differs between patients within a given study [1-4]. Since 2005, exacerbations of IPF identified in our referral centre (Centre Hospitalier Régional de Lille, Lille, France) have been treated with pulses of methylprednisolone followed by pulses of cyclophosphamide [5]. The main goal of the present retrospective study was to evaluate the mortality of exacerbations of IPF treated with this regimen.

Following admission for aggravation of dyspnoea, a series of tests were run to determine diagnosis and functional impairment. When an exacerbation of IPF was diagnosed, patients were treated with a methylprednisolone pulse $(1,000 \mathrm{mg})$ at days 1-3 


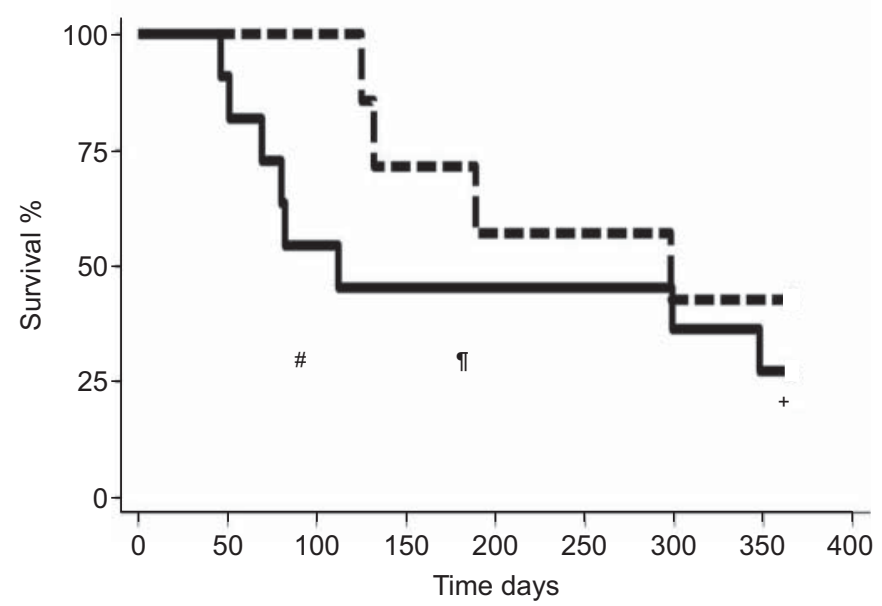

FIGURE 1. Survival in acute exacerbations ( - ) and sub-acute exacerbations (------) of idiopathic pulmonary fibrosis. Survival status at 1 yr was known for all patients; therefore survival curves represent actual data. Differences are expressed by log-rank test at 3 months, 6 months and 1 yr. ${ }^{*}: p=0.05 ;{ }^{\bullet}: p=0.18 ;{ }^{+}: p=0.35$.

and on day 4 placed on an escalating regimen of cyclophosphamide with an initial dose of $500 \mathrm{mg}$ intravenously [5]. The dose of cyclophosphamide was increased by $200 \mathrm{mg}$ every 2 weeks, provided the total white blood cell count remained at $>3,000$ cells $\cdot \mathrm{mm}^{-3}$. The maximum single administered dose was $1,500 \mathrm{mg}$ of cyclophosphamide. The diagnosis of IPF was reassessed for the purpose of this study [6]. AE was defined as an aggravation of dyspnoea within 1 month, with newly developed pulmonary infiltrates on chest computed tomography (CT), after lung infection, heart failure and pulmonary embolism had been ruled out. The definition of a sub-acute exacerbation (SAE) was identical except that the time span from the onset of symptoms to admission was set at 30-90 days. All the data were retrospectively collected from the patients' medical records. Baseline and exacerbation chest CT scans were reviewed by two physicians unaware of the clinical data. New parenchymal opacities on CT at the time of exacerbation were categorised according to the method of AKIRA et al. [7].

Over a 42-month period, 10 patients were treated for 11 episodes of $\mathrm{AE}$, and seven patients were treated for an SAE. Reevaluation of the CT scan was possible in 14 out of the 18 cases. Newly appeared opacities were labelled as peripheral in six $(43 \%)$ cases, multifocal in three cases and diffuse in five $(36 \%)$ cases. Characteristics at baseline and during the exacerbation are presented in table 1 . All patients were alive 1 month after treatment was initiated. At 3 months, $72 \%$ of patients were alive: all patients with SAE and 55\% patients with AE. At 6 months, overall survival was $56 \%$; 1 -yr survival was $33 \%$ (fig. 1 ). The cause of death was respiratory failure in all cases. Forced vital capacity at admission was significantly associated with 6- and 12 -month survival $(\mathrm{p}=0.01$ and $\mathrm{p}=0.003$, respectively). Likewise, a higher arterial oxygen tension $\left(\mathrm{Pa}_{2} \mathrm{O}_{2}\right)$ at admission for exacerbation was associated with better 6-month survival $(p=0.03)$. Patients with a higher body mass index (BMI) had better 1-yr survival. The type of high-resolution CT pattern was not related to survival. Three patients were admitted to the intensive care unit (ICU) for acute respiratory failure during

TABLE 1 Characteristics of patients at baseline and at admission for exacerbation.

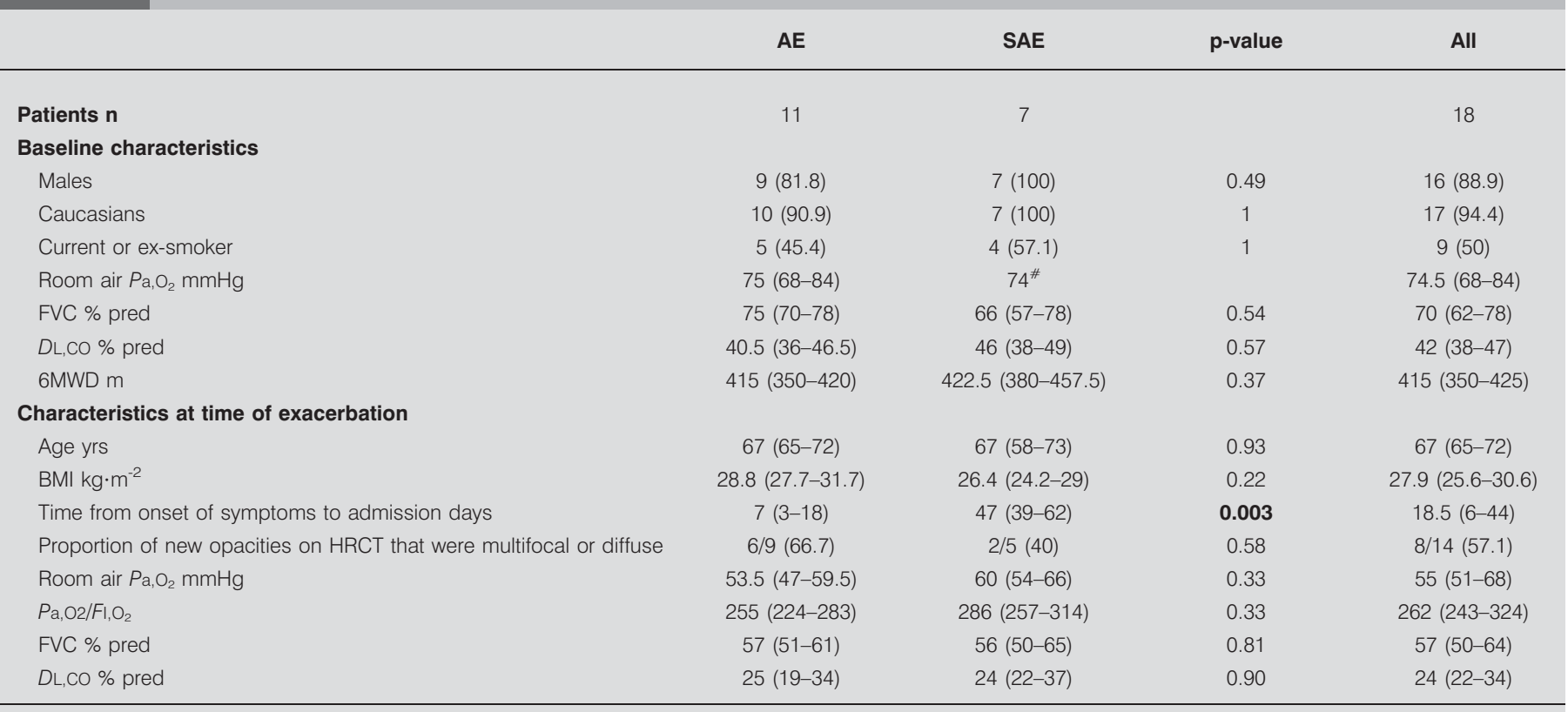

Data are expressed as median (interquartile range) for continuous variables and $\mathrm{n}(\%)$ or $\mathrm{n} / \mathrm{N}(\%)$ for categorical variables, unless otherwise stated. Data and groups were compared using Fisher's exact test and Mann-Whitney U-test. In all cases, two-sided tests were used; p-values $<0.05$ (shown in bold text) were deemed statistically significant. AE: acute exacerbation; SAE: sub-acute exacerbation; $\mathrm{Pa}_{2} \mathrm{O}_{2}$ : arterial oxygen tension; FVC: forced vital capacity; \% pred: \% predicted; DL,CO: diffusion capacity of the lung for carbon monoxide; GMWD: 6-min walking test distance; BMI: body mass index; HRCT: high-resolution computed tomography Fl, $\mathrm{O}_{2}$ : inspired oxygen fraction. ${ }^{*}: n=1$. 
the second or third month of treatment. Two of them were intubated and died at day 1 and day 17 of mechanical ventilation, respectively, from worsening hypoxaemia and multiple organ failure. The third patient was not intubated and died 6 days after ICU admission. While undergoing treatment for IPF exacerbation, six patients received antibiotics for a suspected undocumented respiratory infection; the outcome was positive. Pulmonary infection was suspected in the three patients admitted to the ICU but was documented in only one case. None of these suspected infectious episodes were related to neutropenia, as no severe haematological toxicity was observed. No urological or kidney toxicity was observed.

Our study describes the characteristics and outcome of exacerbations of IPF occurring in a Caucasian population, and treated by a protocol combining pulse steroid therapy followed by pulse cyclophosphamide therapy. Survival appears better than that which has previously been reported, mainly in Asian populations, without systematic immunosuppressive treatment. Most publications report a very high mortality rate, up to $85 \%$. Most deaths occur within a few weeks after the onset of the exacerbation. A recent review estimated the mortality rate at 1 month to be $60 \%$ (70\% at 3 months) and the in-hospital mortality rate was recently reported at 50\% [1]. In our study, mortality appears to be lower, and to occur later. These differences may be explained by the efficiency of the cyclophosphamide treatment regimen, but also by differences in the studied populations or by an over-estimation of mortality in previously published studies. In most observations, patients are treated with corticosteroids (often by 500-1,000-mg methylprednisolone pulses), sometimes with the addition of immunosuppressive therapies. Some authors report better survival in patients treated with cyclosporin A than in patients treated with corticosteroids alone, suggesting that immunosuppressive therapy associated with corticosteroids could be more effective than corticosteroids alone. There are currently no trials of antioxidant or anti-fibrotic drugs for AEs of IPF. Treatment of stable IPF with pirfenidone was not found to be associated with a lesser occurrence of AEs [8]. One study, conducted on a limited number of patients, showed that anticoagulant therapy reduces the mortality of acute exacerbations [9]. Optimal treatment for exacerbations of IPF has yet to be defined.

Most publications about AEs of IPF come from East Asia (South Korea and Japan). European and North American publications are case series on selected patients (e.g. with a lung biopsy), with potentially biased mortality rates. Genetic and/or environmental differences between populations could influence the incidence, expression and severity of AEs of IPF. Mortality in pathological studies can also be biased, as the morbiditymortality of the lung biopsy adds to the mortality of the exacerbation. The mortality rates from prospective studies are among the lowest reported. This could be explained by a better identification and inclusion of less severe cases, the incidence of exacerbations also being higher in these studies.

The association found between forced vital capacity and $\mathrm{Pa}, \mathrm{O}_{2}$ at admission for exacerbation and survival suggests that the prognosis of exacerbations might be linked to their initial severity as well as to the severity of IPF $[1,10]$ and the $\mathrm{Pa}_{1} \mathrm{O}_{2} /$ inspired oxygen fraction ratio seems to be lower in the studies reporting the highest mortality rates. A higher BMI was found to be a protecting factor towards 1-yr mortality. Obesity has been shown to be a factor in favour of good prognosis in stable IPF. In our study, survival was not related to CT findings.

We also studied SAEs of IPF, along with AEs, as we found that seven patients with IPF had a similar clinical and radiological presentation, developed over 4-12 weeks. SAEs of IPF have not been addressed in previous publications. We found no difference in epidemiological and functional data at baseline and during exacerbation. Survival analysis at 3 months showed that mortality was significantly higher in AEs, but 1-yr survival was similar in the two groups. These data suggest that SAEs of IPF could be a slower-evolving type of the same underlying pathophysiological mechanism as AEs, resulting in a similar medium-term prognosis.

\section{E. Morawiec*, I. Tillie-Leblond*, V. Pansini", J. Salleron", M. Remy-Jardin" and B. Wallaert*}

*Clinique des Maladies Respiratoires, Centre de Compétence des Maladies Pulmonaires Rares, Hôpital Calmette, Centre Hospitalier Régional Universitaire (CHRU) de Lille and Université de Lille 2, "Service d'Imagerie Thoracique, Hôpital Calmette, CHRU de Lille, and 'Service de Biostatistiques, Centre d'Etudes et de Recherche en Informatique Médicale, CHRU de Lille, Lille, France.

Correspondence: B. Wallaert, Clinique des Maladies Respiratoires, Hôpital Albert Calmette, Bd du Pr Leclercq, 59037 Lille cedex, France. E-mail: benoit.wallaert@chru-lille.fr

Statement of Interest: A statement of interest for I. Tillie-Leblond can be found at www.ersjournals.com/site/misc/statements. xhtml

\section{REFERENCES}

1 Song JW, Hong S-B, Lim C-M, et al. Acute exacerbation of idiopathic pulmonary fibrosis: incidence, risk factors and outcome. Eur Respir J 2011; 37: 356-363.

2 Collard HR, Moore BB, Flaherty KR, et al. Acute exacerbations of idiopathic pulmonary fibrosis. Am J Respir Crit Care Med 2007; 176: 636-643

3 Agarwal R, Jindal SK. Acute exacerbation of idiopathic pulmonary fibrosis: a systematic review. Eur J Intern Med 2008; 19: 227-235.

4 Hyzy R, Huang S, Myers J, et al. Acute exacerbation of idiopathic pulmonary fibrosis. Chest 2007; 132: 1652-1658.

5 Baughman RP, Lower EE. Use of intermittent, intravenous cyclophosphamide for idiopathic pulmonary fibrosis. Chest 1992; 102: 1090-1094.

6 American Thoracic Society, European Respiratory Society. Idiopathic pulmonary fibrosis: diagnosis and treatment. International consensus statement. Am J Respir Crit Care Med 2000; 161: 646-664.

7 Akira M, Kozuka T, Yamamoto S, et al. Computed tomography findings in acute exacerbation of idiopathic pulmonary fibrosis. Am J Respir Crit Care Med 2008; 178: 372-378.

8 Taniguchi H, Ebina M, Kondoh Y, et al. Pirfenidone in idiopathic pulmonary fibrosis. Eur Respir J 2010; 35: 821-829.

9 Kubo H, Nakayama K, Yanai M, et al. Anticoagulant therapy for idiopathic pulmonary fibrosis. Chest 2005; 128: 1475-1482.

10 Nishiyama $\mathrm{O}$, Taniguchi $\mathrm{H}$, Kondoh $\mathrm{Y}$, et al. A simple assessment of dyspnoea as a prognostic indicator in idiopathic pulmonary fibrosis. Eur Respir J 2010; 36: 1067-1072. 